\title{
Givinostat reduces adverse cardiac remodeling through regulating fibroblasts activation
}

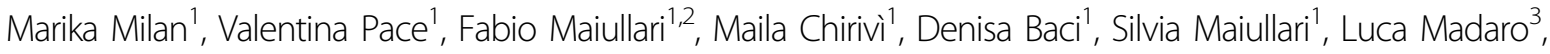 \\ Sonia Maccari ${ }^{4}$, Tonino Stati ${ }^{4}$, Giuseppe Marano ${ }^{4}$, Giacomo Frati ${ }^{5,6}$, Pier Lorenzo Puri ${ }^{7}$, Elena De Falco $\mathbb{B}^{5}$, \\ Claudia Bearzi ${ }^{1}$ and Roberto Rizzi, ${ }^{1,2}$
}

\begin{abstract}
Cardiovascular diseases (CVDs) are a major burden on the healthcare system: indeed, over two million new cases are diagnosed every year worldwide. Unfortunately, important drawbacks for the treatment of these patients derive from our current inability to stop the structural alterations that lead to heart failure, the common endpoint of many CVDs. In this scenario, a better understanding of the role of epigenetics - hereditable changes of chromatin that do not alter the DNA sequence itself - is warranted. To date, hyperacetylation of histones has been reported in hypertension and myocardial infarction, but the use of inhibitors for treating CVDs remains limited. Here, we studied the effect of the histone deacetylase inhibitor Givinostat on a mouse model of acute myocardial infarction. We found that it contributes to decrease endothelial-to-mesenchymal transition and inflammation, reducing cardiac fibrosis and improving heart performance and protecting the blood vessels from apoptosis through the modulatory effect of cardiac fibroblasts on endothelial cells. Therefore, Givinostat may have potential for the treatment of CVDs.
\end{abstract}

\section{Introduction}

Cardiac remodeling and fibrosis are compensatory mechanisms consequent to ischemic events ${ }^{1}$ and they strictly determine the clinical outcome. Indeed, after an ischemic event there is an initial phase of remodeling and recovery, during which damaged cardiomyocytes (CMs) are replaced by new cells; however, this leads to a secondary phase characterized by fibrosis ${ }^{2}$, a process that, when unchecked, causes the generation of excessive remodeling of the cardiac extracellular matrix, oxidative stress, and inflammation within the ischemic microenvironment ${ }^{3}$. Although fibrosis and

\footnotetext{
Correspondence: Claudia Bearzi (claudia.bearzi@cnr.it) or

Roberto Rizzi (roberto.rizzi@cnr.it)

${ }^{1}$ Institute of Cell Biology and Neurobiology (IBCN), National Research Council of Italy (CNR), Monterotondo Scalo, Rome 00015, Italy

2Operational Research Unit, Fondazione di Ricerca e Cura Giovanni Paolo II, Largo Gemelli 1, Campobasso, Italy

Full list of author information is available at the end of the article Edited by A. Stephanou
}

inflammation are initially beneficial ${ }^{4}$, they become detrimental in the long term, suggesting that therapy should aim for the control rather than the suppression of both events.

Among the biological and molecular mechanisms involved in the adaptive response to a cardiac insult, histone deacetylase (HDAC)-mediated epigenetics processes are receiving a special attention. HDACs are common enzymes regulating deacetylation of core histones and are strictly correlated to the regulation of homeostatic gene expression of vascular and cardiac cell populations, including stem cell commitment ${ }^{5}$. More importantly, abnormal acetylation of core histones, a process likely linked to environmental factors, has been associated with major cardiovascular diseases ${ }^{6}$. After a cardiac insult, HDACs activity is enhanced, resulting in increased proliferation, migration, and apoptosis of adventitial fibroblasts (FBs), endothelial cells (ECs), and muscle cells, as well as stimulation of macrophage (MP) 
activation and phenotype switching ${ }^{7}$ suggesting an involvement of HDACs in driving the response to injury and remodeling even through the early inflammatory phase. A wide range of molecules have been tested in their ability to inhibit HDACs ${ }^{8}$. Pan- and selective HDAC inhibitors (HDACi) have been shown to preserve cardiac function in disease states by exerting an antiinflammatory effect and reducing cardiac hypertrophy and fibrosis ${ }^{9,10}$ through signals mainly targeting oxidases and/or specific kinases ${ }^{11,12}$. Despite this, epigeneticsbased therapies are still limited in the cardiovascular field and the use of the HDACi has still to be clearly elucidated, including safety and long-term effects.

Givinostat (ITF2357) is a powerful pan-HDACi that has gained considerable attention due to its varied applicability, efficacy, and safety in humans. Described in $2005^{13}$, Givinostat is currently being tested in clinical trials on different diseases ${ }^{14-18}$. The drug has been shown to decrease tnf- $\alpha$, il- 6 , and il- 1 levels, producing a striking reduction of the inflammatory response in combination with pro-angiogenic effects. To date, the effects of Givinostat on cardiac diseases remain to be verified, but studies on Duchenne muscular dystrophy (DMD) suggest that the HDACi might act beneficially on the cardiac muscle as well ${ }^{18}$.

Therefore, we decided to study the biological and functional efficacy of Givinostat on acute myocardial infarction (AMI). We found that the drug improved post-AMI heart function by hindering the development of fibrosis, likely via a mechanism targeting endothelialto-mesenchymal transition (EndMT). Thus, Givinostat holds promise for the treatment of cardiovascular diseases.

\section{Results}

To test the efficacy of Givinostat on heart failure, 10week-old C57 mice underwent surgery to induce AMI by permanent ligation of the left descending coronary artery: one group of mice was treated daily with Givinostat for 1, 3, 7,15 , or 30 days, while a control group was administered with saline. At the end of the treatments, mice were killed.

Cardiac performance was evaluated by echocardiography. Saline administered mice suffered progressive declines in fractional shortening (FS) as expected (Fig. 1a). Interestingly, Givinostat treatment significantly improved the percentage of FS at day 7, 15, and 30 (Fig. 1a) compared to controls. Left Ventricular End Diastolic Volume (LVEDV), Left Ventricular End Systolic Volume (LVESV), Left Ventricular End Diastolic Diameter (LVEDD), and wall thickness (WT) measurements confirmed modulation of cardiac remodeling. There were no differences in the WT parameter, which was calculated as Frontal Wall thickness+Posterior Wall thickness/2, between the two groups at day 30 . Indeed, the hypertrophy of the back wall of control animals is counteracted by the reduced loss of muscle tissue in treated group (Fig. 1a; Table 1).

Notably, Givinostat administration modified the epigenetic state of cardiac tissue, by increasing protein level of acetylated Histone 3 at day 3, 7, 15, and 30 (Fig. 1b). The improvement in the percentage of FS, in treated animals, was histologically accompanied by a decreased percentage of cardiac fibrotic area (Masson's trichrome) over the time respect to controls (Fig. 1c).

Coherently, quantitative RT-PCR (qRT-PCR) analysis showed a diminished expression of fibrosis-associated genes, including collagen 1a1 (Col1a1), collagen 1a2 (Col1a2) and collagen $3 \mathrm{a} 1$ (Col3a1) in the hearts of Givinostat treated mice.

Moreover, inflammatory activity was drastically reduced following treatment with the HDACi. Indeed, the expression of interleukins il- $1 \alpha$ and il- $1 \beta$ (Fig. $2 a$ ) was significantly decreased one day after Givinostat administration, leading us to speculate that the beneficial effects of the drug may act via modulation of inflammatory cells, such as macrophages (MPs). Expressions of tumor necrosis factor- $\alpha$ (tnf- $\alpha$ ) and $\mathrm{f} 4 / 80$ (a macrophage receptor) were significantly reduced in infarcted hearts treated with Givinostat. This outcome was confirmed by histological measurements, where F4/80 and MMP9 positive areas were detected and normalized to the total cardiac section (Fig. 2c).

The above results suggest that the beneficial effect of Givinostat depends, at least partially, on the blunting of the inflammatory process.

We assumed that the cells mediating this HDACi effect were MPs. To test this hypothesis, we isolated the MP population from the bone marrow of mice. One MP group was treated with Lipopolysaccharide (LPS) and another with Interleukin 4 (IL-4) to induce respectively M1 and M2 phenotypes. A third MP group was non-polarized (Naive). All clusters were treated with Givinostat and analyzed after $24 \mathrm{~h}$ by qRT-PCR.

The expression of mmp9, a metalloproteinase enhancing migration capacity of MPs in M1 phase through the recall sites, was significantly reduced (Fig. 2d), while, in the M2 phenotype the expression of bcl2, which in MPs guarantees protection from apoptosis (Fig. 2d), was increased after treatment. Givinostat administration in presence of AMI did not alter body and heart weights over the time (Fig. 2e) compare to the control.

In addition, Givinostat treatment significantly increased placenta growth factor (plgf) gene expression, a known angiogenic factor, which in M2 recalls blood vessels to support the regenerative phase (Fig. 2d). Lastly, bone morphogenic protein 2 (bmp-2) increased expression (Fig. 2d) could robustly activate MPs through pSmad1/5/ 8 signaling pathway generating a positive feedback loop by increasing the expression of angiogenic factors. Data were confirmed by histological analysis (Fig. 2e). 
$\underline{A}$ Echocardiographic analysis
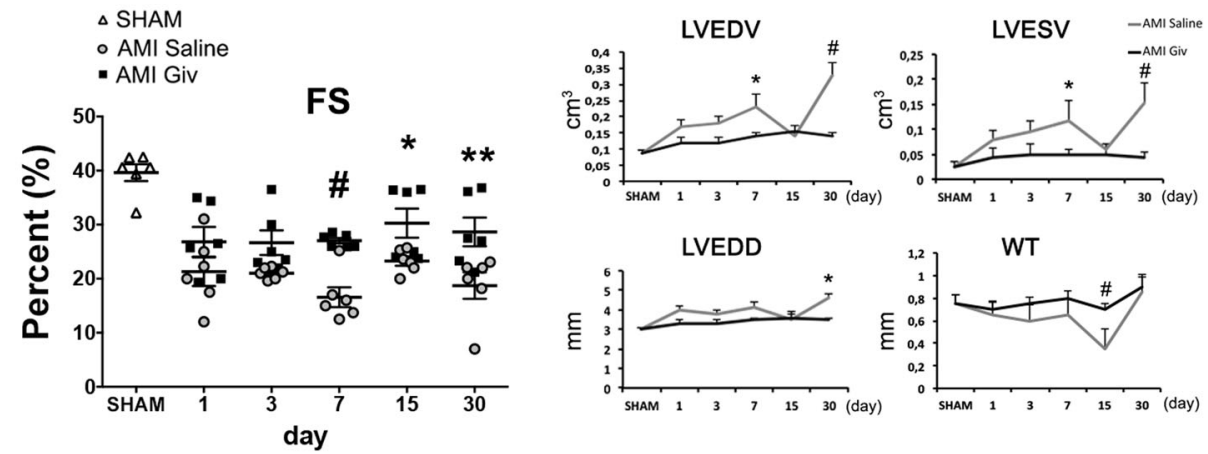

SHAM

AMI Saline

AMI Giv
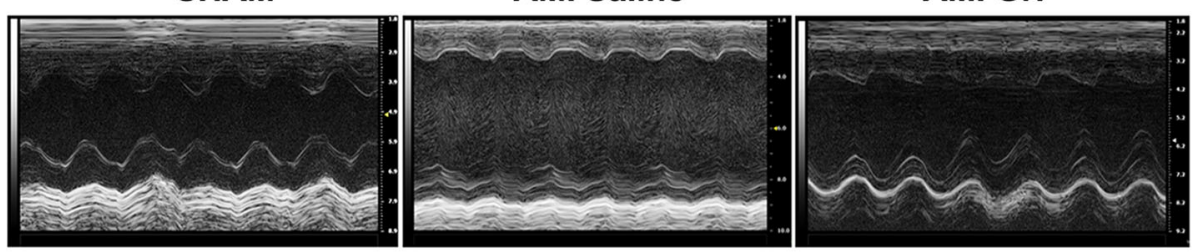

B

Acetylation
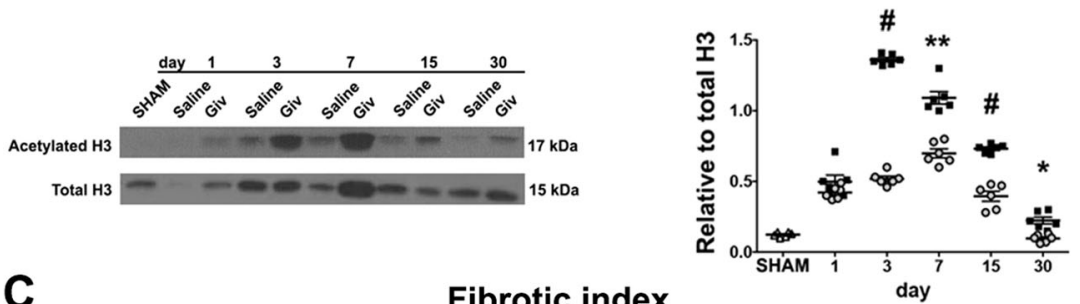

$\underline{\text { C }}$

Fibrotic index
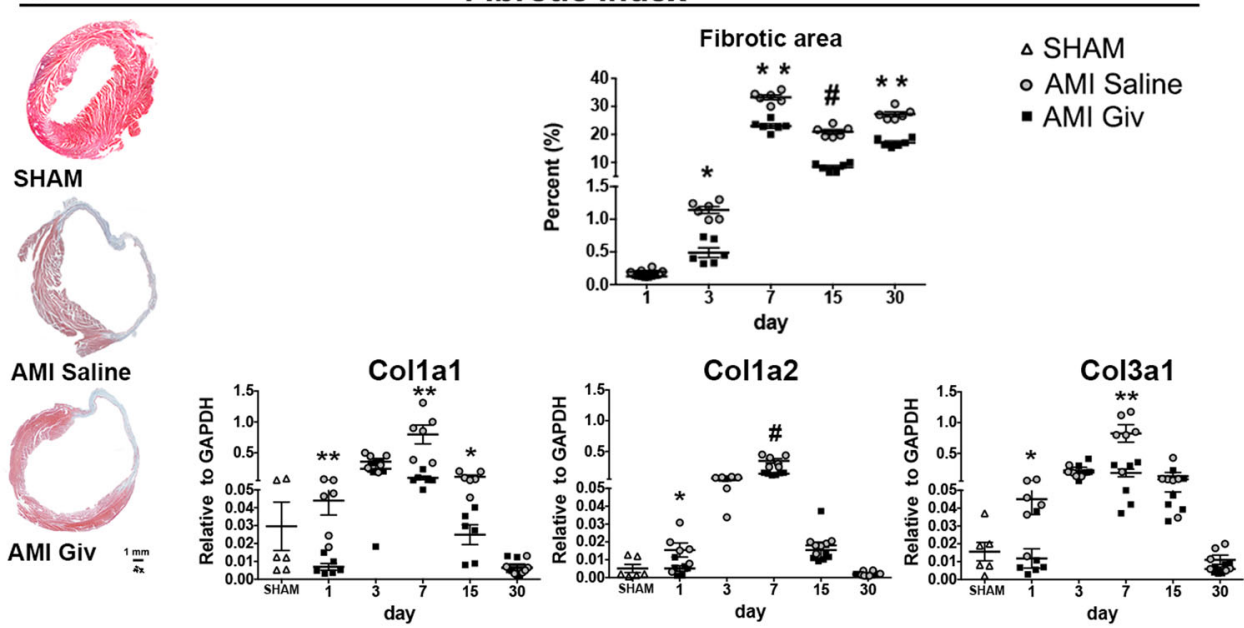

Fig. 1 Givinostat effect on infarcted heart and cardiac fibrosis. a Echocardiographic measurements indicate an amelioration in fractional shortening (FS), left ventricular end diastolic volume (LVEDV), left ventricular end systolic volume (LVESV), left ventricular end diastolic diameter (LVEDD) and wall thickness (WT). Representative M-mode images of control mice (left panel) and after injection with saline (middle panel) or Givinostat (right panel) in AMI mice. b Western blot analysis shows an increased acetylated H3 protein levels in the whole heart tissue after Givinostat administration compared to saline group. c After Givinostat treatment, four sections per mouse were analyzed by Masson's trichrome staining. Scale bar represent $1 \mathrm{~mm}$. Percentage of fibrotic area: fibrotic area/total area $\times 100$. Quantitative RT-PCR illustrates the Givinostat effect on the expression of fibrosis-related genes (Col1a1, Col1a2 and Col3a1) at different time points. $N=6$ mice for time point. Error bars represent \pm SEM. Student's $t$ test, ${ }^{*} p<$ $0.05,{ }^{* *} p<0.01,{ }^{\#} p<0.001$ 


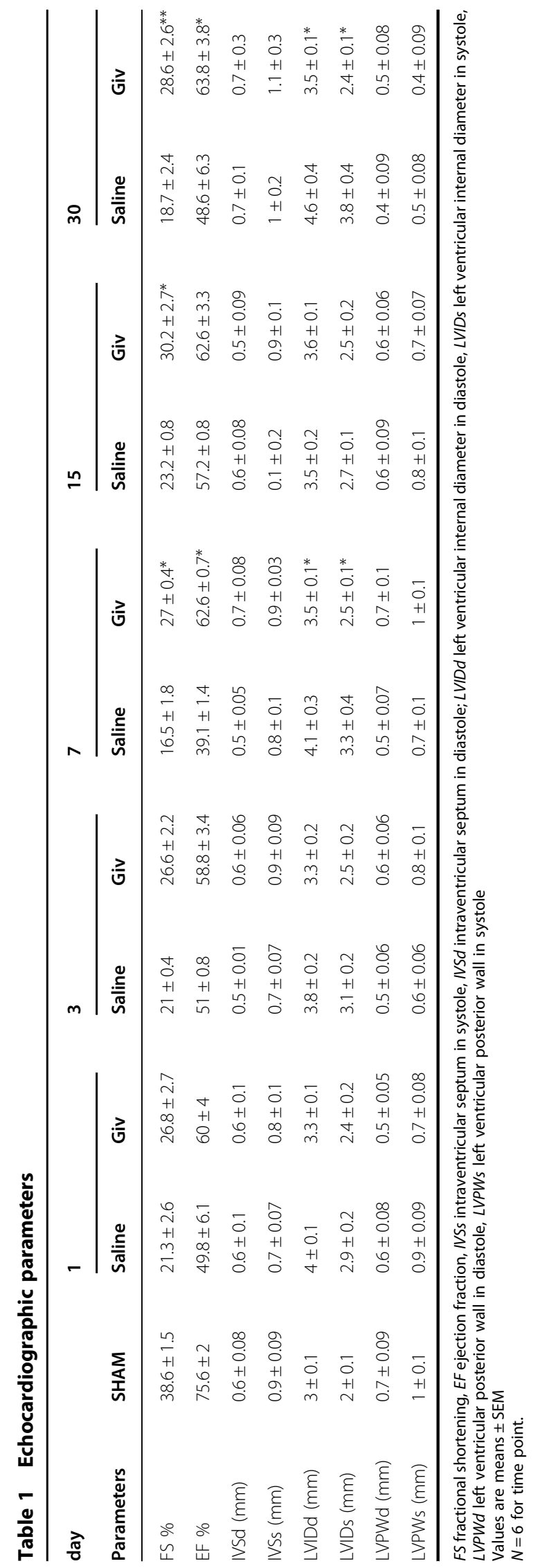

Hence, we isolated primary MPs and CMs from the C57 neonatal mice to perform co-culture experiments to assess the effect of the latter on the former (Supplementary Figure 1). The in vitro experiment involved the gene expression analysis of CMs alone or co-cultured with the MPs-naive or -M1 or -M2 phenotypes after Givinostat administration. MPs-M2 exposed to Givinostat increased the maturation of neonatal CMs, suggesting that MPs can influence $\mathrm{CM}$ fate. Moreover, a very interesting effect consisted in the reduction of the Caspase 3 (casp3) expression, which hints a protective role from apoptosis confirmed by bcl 2 expression. In contrast, direct exposure of CMs to Givinostat resulted in induction of early cardiac genes implying a re-activation of the cardiac embryonic program, and suggesting the triggering of the hypertrophic pathway, which is prevented if the effect of Givinostat is mediated by the MPs-M2.

We also investigated the relationship between MPs and cardiac FBs using the same co-culture model (Supplementary Figure 1). Givinostat had a direct action in FB cultures, increasing the expression of bone morphogenic protein 7 (bmp-7), known to be capable of inhibiting the action of TGF- $\beta$ in triggering EndMT ${ }^{19,20}$ and decreasing p53 expression. Indeed, p53 inhibition promotes cardiac angiogenesis and reduces heart dysfunction induced by pressure damage and adaptive hypertrophy post- $\mathrm{AMI}^{21}$. Snail- 1 and -2 , which are directly regulated by tgf- $\beta$ in EndMT response, were downregulated when FBs were cocultured with MPs-M1. Finally, reduction of $\alpha$-sma and increased e-cadherin expressions indicate that mesenchymal to endothelial transition (MET) was taking place, a process regulated by bmp-7. Importantly, the effect of Givinostat changes depending on whether the cells are stimulated directly or when co-cultured with MPs. In case of direct MPs-Givinostat stimulation M2 phenotype is preferred. This data confirms what is already known in the literature with reference to the wide spectrum of HDACi effects ${ }^{22}$. While the MP-mediated effect on CMs regards apoptosis protection ( $\mathrm{bcl} 2)$, this outcome is completely canceled if the stimulation is direct on CMs without the involvement of the inflammatory compartment. Protection against apoptosis in the CMs compartment was also confirmed in vivo, after infarction, by immunofluorescence experiments. Figure $3 a$ shows that the positivity of CMs for TUNEL was reduced in the hearts of Givinostat treated animals at the early time points (Fig. 3a). Subsequently, after 15 and 30 days, the prevention decreased bringing the ratio similar to the control. The same effect in preventing the activation of the $\mathrm{CM}$ embryonic program ( $\beta$-mhc, anf, gata 4$)$ in vitro, was also confirmed in vivo. We measured the crosssectional area (CSA) of CMs both in the border and in the contralateral zones and we confirmed that the cardiac 


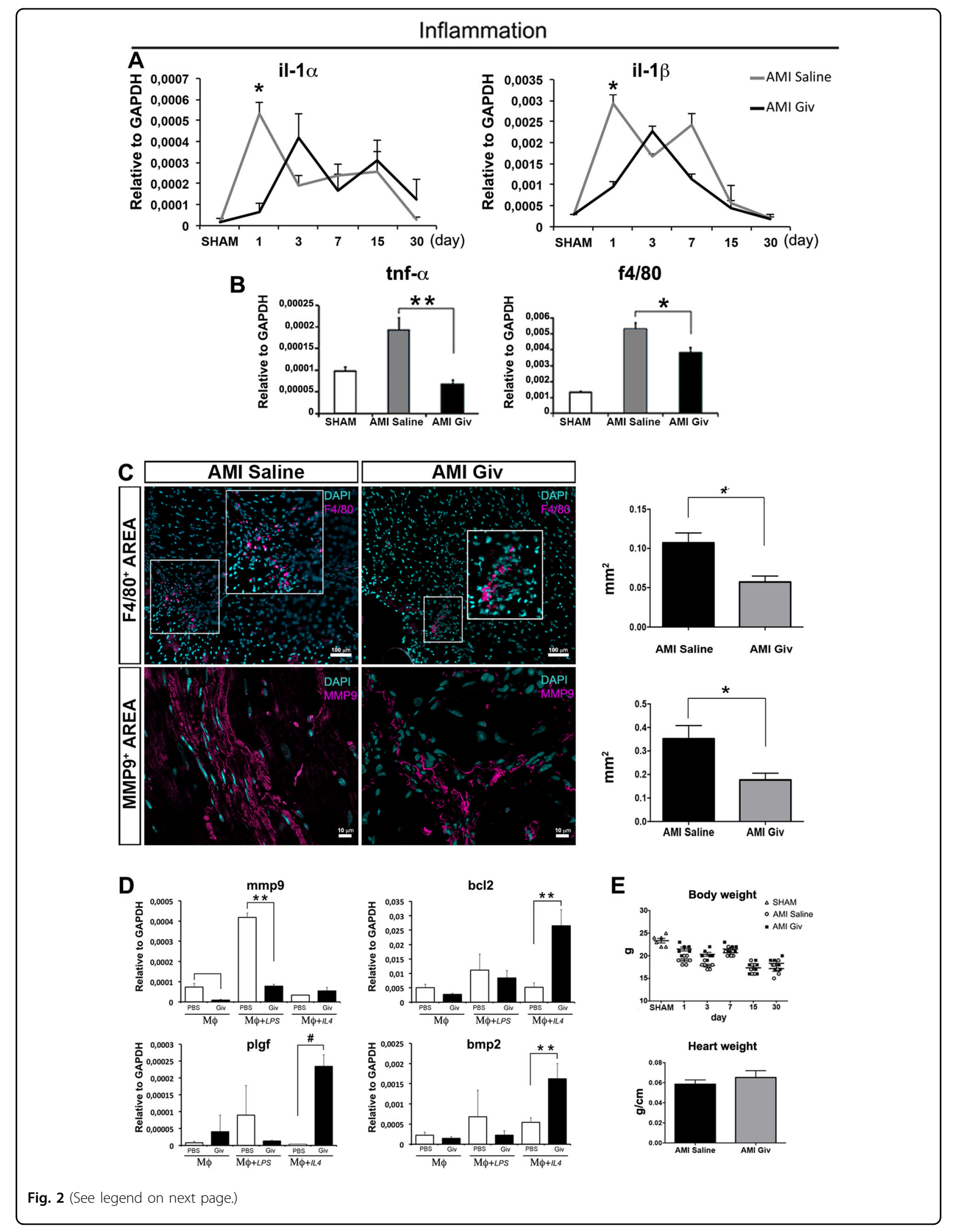


(see figure on previous page)

Fig. 2 Inflammatory process assays. a The graphs display the mRNA levels of interleukins il-1a and il- $\beta$ after Givinostat administration in infarcted hearts. $\mathbf{b}$ Expression levels of tnf- $a$ and f4/80 genes in AMI mice treated with Givinostat after 1 day. c Sections of AMl mouse heart treated with saline (left panels) and Givinostat (right panels) at day 7 composed of cells positive for F4/80 (magenta, upper images) and MMP9 (magenta, lower images). Scale bars represent 100 and $10 \mu \mathrm{m}$ respectively. The graphs highlight F4/80 and MMP9 infiltrating areas ( $\mathrm{mm}^{2}$ ) normalized to the total area of cardiac tissue, in the animals treated with Givinostat and in the control group. $\mathbf{d}$ Quantitative RT-PCR demonstrates a reduction of mmp9 expression in M1 macrophages and an overexpression of bcl2, plgf and bmp2 genes in M2 macrophages after Givinostat exposure. e Effect of Givinostat on body weight at different time points and on heart weight normalized to the tibia length, at the end of the treatment in AMl mice. $N=6$ mice for time point. Error bars represent \pm SEM. Student's $t$ test, ${ }^{*} p<0.05,{ }^{* *} p<0.01,{ }^{*} p<0.001$

hypertrophy program was strongly reduced in the Givinostat treated group (Fig. 3b).

Interestingly, the Givinostat treatment significantly reduced the whole number of apoptotic cells in the border zone starting from day 3 up to 1 month (Fig. 3c). Histological analysis of AMI mice treated with Givinostat, also displayed an enhanced vasculature due to an increased number of $\alpha$-SMA positive vessels/total area $\left(\mathrm{mm}^{2}\right)$ at all time points (Fig. 3d) in combination with a parallel increasing in capillary density both in the border and in the contralateral zones (Fig. 3e). Another result supporting the Givinostat protective role is the increased Connexin 43 expression (CX43) in treated animals (Fig. 3f), also confirmed by western blot (Fig. 3h).

Surprisingly, we noted that many of the vessels present in the hearts of the Givinostat treated animals were preserved from apoptosis after AMI, that, instead, was present in the surrounding tissue (Fig. 3g). Moreover, we observed a reduction of EndMT-related markers including a decreased gene expression of tgf- $\beta$, twist 1 and 2 and snail 1 and 2 and increase of bmp-7, known to counteract the fibrogenic activity of tgf- $\beta$ (Fig. $4 \mathrm{a})^{20}$. Coherently, TGF- $\beta$ protein expression, a key regulator of cardiac fibrosis ${ }^{23}$, were significantly reduced compared to controls at $\mathrm{d} 7$ by Givinostat (Fig. 4b). A similar decrease was observed in mmp9, a pro-fibrotic marker equally involved in the pathological cardiac remodeling (Fig. 4a). Remarkably, the solely injection of Givinostat in the absence of AMI did not alter the gene expression levels of pro-fibrotic markers (supplementary Figure 2).

We than moved to an in vitro human model; human FBs were starved for $6 \mathrm{~h}$ and subsequently, treated with $50 \mathrm{nM}$ Givinostat and exposed to $18 \mathrm{~h}$ of hypoxia at $1 \%$ oxygen, together with the PBS control group (Fig. 5a). The day after, cells were fixed in 4\% PFA and stained for Ki67 to evaluate the proliferative capacity. The Givinostat group showed a proliferative index higher than the control, following stressful conditions (Fig. 5b). We performed a Chromatin Immuno-precipitation (ChIP) assay to detect protein-DNA interactions, among acetylation of histone 3 (H3Ac) with its target genes. The identification of the target genes and the mechanisms, by which transcription factors control gene expression, are necessary to direct the way of investigation. Specific DNA sequences were also examined by PCR. Surprisingly, we found that bmp-7, nos3, and e-cadherin ${ }^{24}$ promoters were associated with H3Ac confirming the active status of endothelial promoters following the treatment with Givinostat (Fig. 5c). qRT-PCR confirmed the upregulation of others endothelial genes (plgf, e-cad); in contrast, typical FB genes ( $\mathrm{n}$-cad, twist1 and 2, snail1, fibronectin, mmp9) were downregulated (Fig. $5 \mathrm{~d}$ ). Tgf- $\beta$, bmp- 2 and -4 were also downregulated in the first $24 \mathrm{~h}$ while bmp- 7 was more expressed making us speculate that the TGF- $\beta$ mediated EndMT mechanism was inhibited (Fig. 5d). Finally, we counted the double co-localization for Vimentin (FBs) and vWF (ECs) as transdifferentiation index.

Following hypoxic condition, FBs should activate EndMT mechanism; unexpectedly they triggered the reverse pathway known as MET after Givinostat treatment, co-expressing Vimentin and vWF in $\sim 20 \%$ of FBs, compared to the $5 \%$ of the control (Fig. 5e).

We then investigated the effect of Givinostat on human umbilical vein endothelial cells (HUVECs), which were serum-starved for $6 \mathrm{~h}$ and then exposed to hypoxia for 18 h. Givinostat was added to the cell cultures for the same time interval, while the control group was treated with PBS. Direct exposure to Givinostat does not alter the expression of tgf- $\beta$ or bmp-7 in HUVECs (Supplementary Figure 3). Very interesting, however, it is the apoptosis protection offered by Givinostat treatment to ECs, revealed by increased bcl 2 and reduced casp 3 expressions (Supplementary Figure 3). Further, angiogenesis assay was performed with HUVECs exposed to supernatant harvested from starved and hypoxic FBs treated with Givinostat or PBS. Givinostat treated FBs supernatant increased HUVECs angiogenesis compared to the HUVECs directly exposed to Givinostat (Supplementary Figure 4). We performed also perturbation studies pretreating HUVECs with Noggin $(50 \mathrm{ng} / \mathrm{ml})$ or BMP7 (10 $\mathrm{ng} / \mathrm{ml}$ ) and exposing them to supernatant of starved and hypoxic FBs treated with Givinostat or PBS. Noggin is a specific BMP-7 inhibitor that opposes its action in several molecular pathways included EndMT. Supernatant of FBs, starved for $6 \mathrm{~h}$ and exposed to $18 \mathrm{~h}$ of $1 \%$ oxygen 


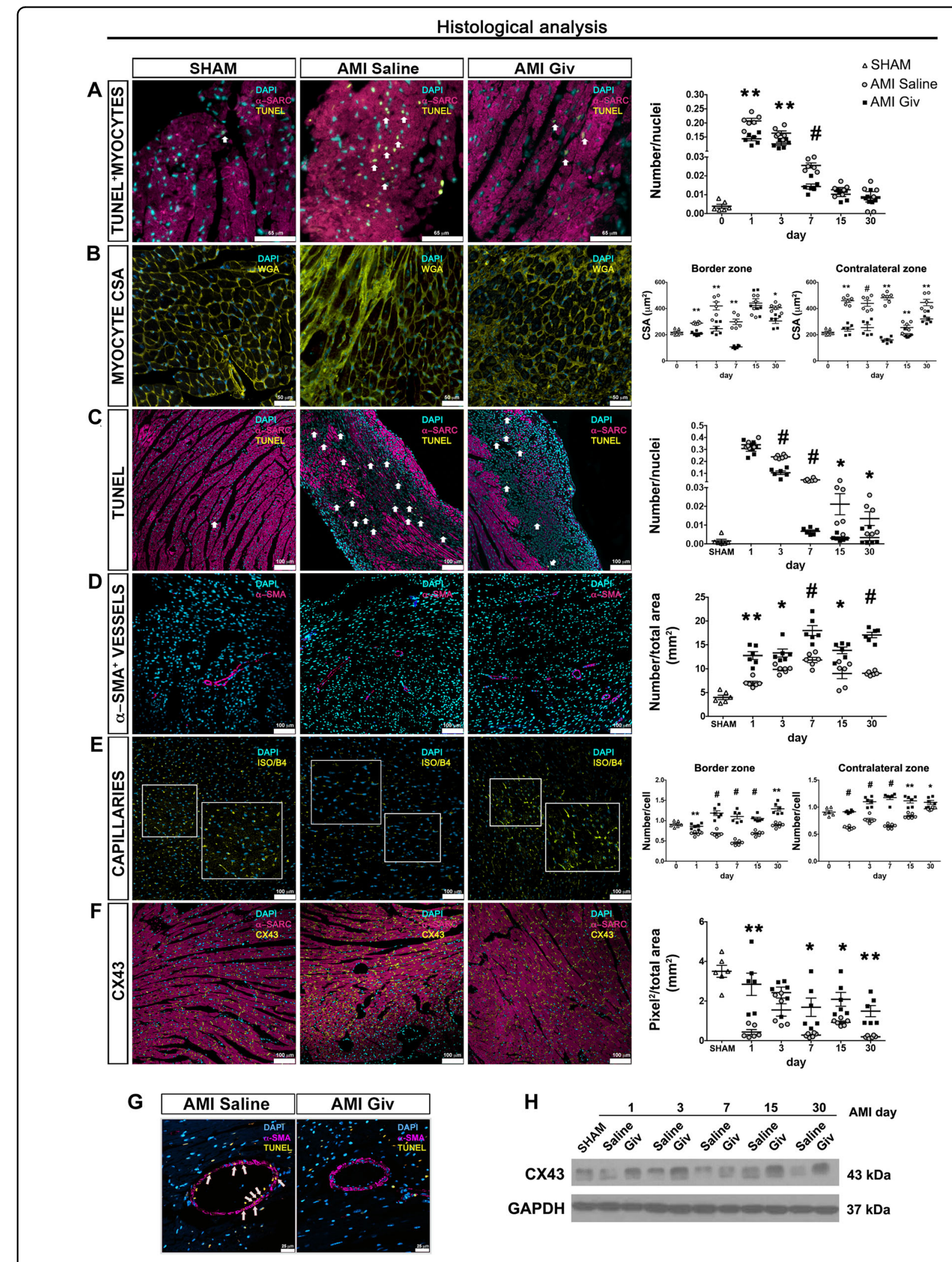

Fig. 3 (See legend on next page.) 
(see figure on previous page)

Fig. 3 Histological analysis. a Apoptotic cardiomyocytes (a-SARC, magenta) were detected by TUNEL labeling (yellow) in SHAM and AMI mice at day 7. Scale bar represent $65 \mu \mathrm{m}$. The graph illustrates the ratio between the number of double positive cells (a-SARC ${ }^{+}$and TUNEL ${ }^{+}$cells) and the nuclei at different time points. b Cross sectional area (CSA) in the border zone at day 30 stained with wheat germ agglutinin (WGA, yellow). Scale bar represent $50 \mu \mathrm{m}$. Plot indicates myocytes $\mathrm{CSA}\left(\mu \mathrm{m}^{2}\right)$ in both the border and the contralateral zone at different time points. c Apoptotic cells were detected by TUNEL labeling (yellow) at day 7. Scale bar represent $100 \mu \mathrm{m}$. The graph indicates the ratio between the number of whole TUNEL positive cells and the nuclei at different time points. $\mathbf{d}$ a-SMA positive vessels labeled in magenta after 30 days. Scale bar represent $100 \mu \mathrm{m}$. The corresponding diagram shows the ratio number of a-SMA positive vessels on total area $\left(\mathrm{mm}^{2}\right)$ at different time points. e Isolectin B4 positive capillaries (yellow) in the border zone at day 30. Scale bar represent $100 \mu \mathrm{m}$. Graphs explain the proportion number of ISO/B4 positive capillaries/cell number in the border and the contralateral zone at different time points. $\mathbf{f}$ Staining for Cx43 (yellow) in the border zone of the infarcted area at day 30. Scale bar represent $100 \mu \mathrm{m}$. Cx43 quantification, expressed as pixel ${ }^{2} /$ total area, is elucidated in the side chart. For Cx43 quantification method details see Materials and methods section and supplementary Figure S5. g Heart cross section of vessel composed of smooth muscle cells (a-SMA, magenta) and apoptotic cells (yellow) highlighted by arrows (white). Scale bar represent $25 \mu \mathrm{m}$. $\mathbf{h}$ Western blot analysis for Cx43 in AMl Givinostat mice vs. AMI Saline group at different time points. $N=6$ mice for time point. Error bars represent \pm SEM. Student's $t$ test, ${ }^{*} p<0.05,{ }^{* *} p<0.01,{ }^{*} p<$ 0.001

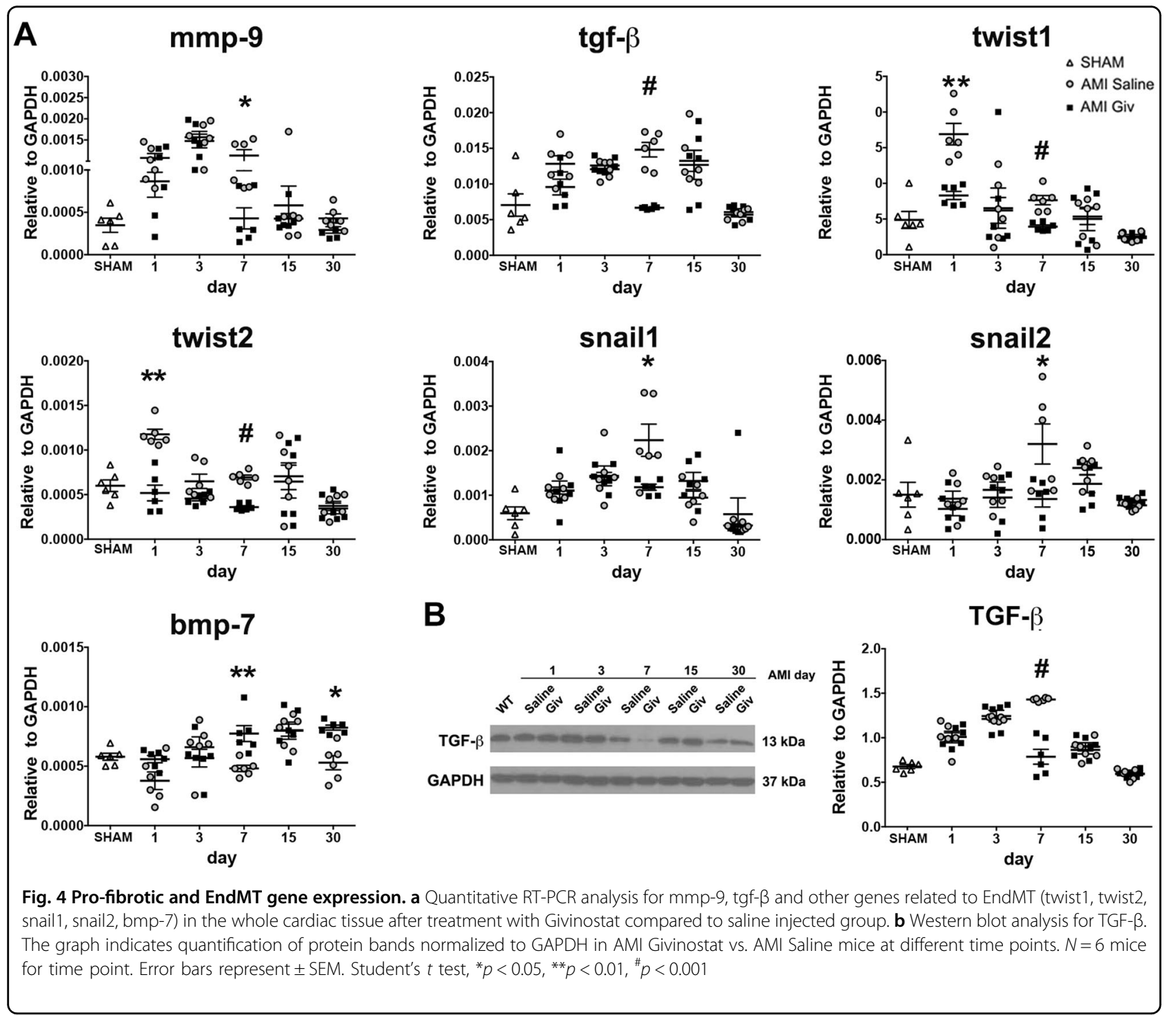




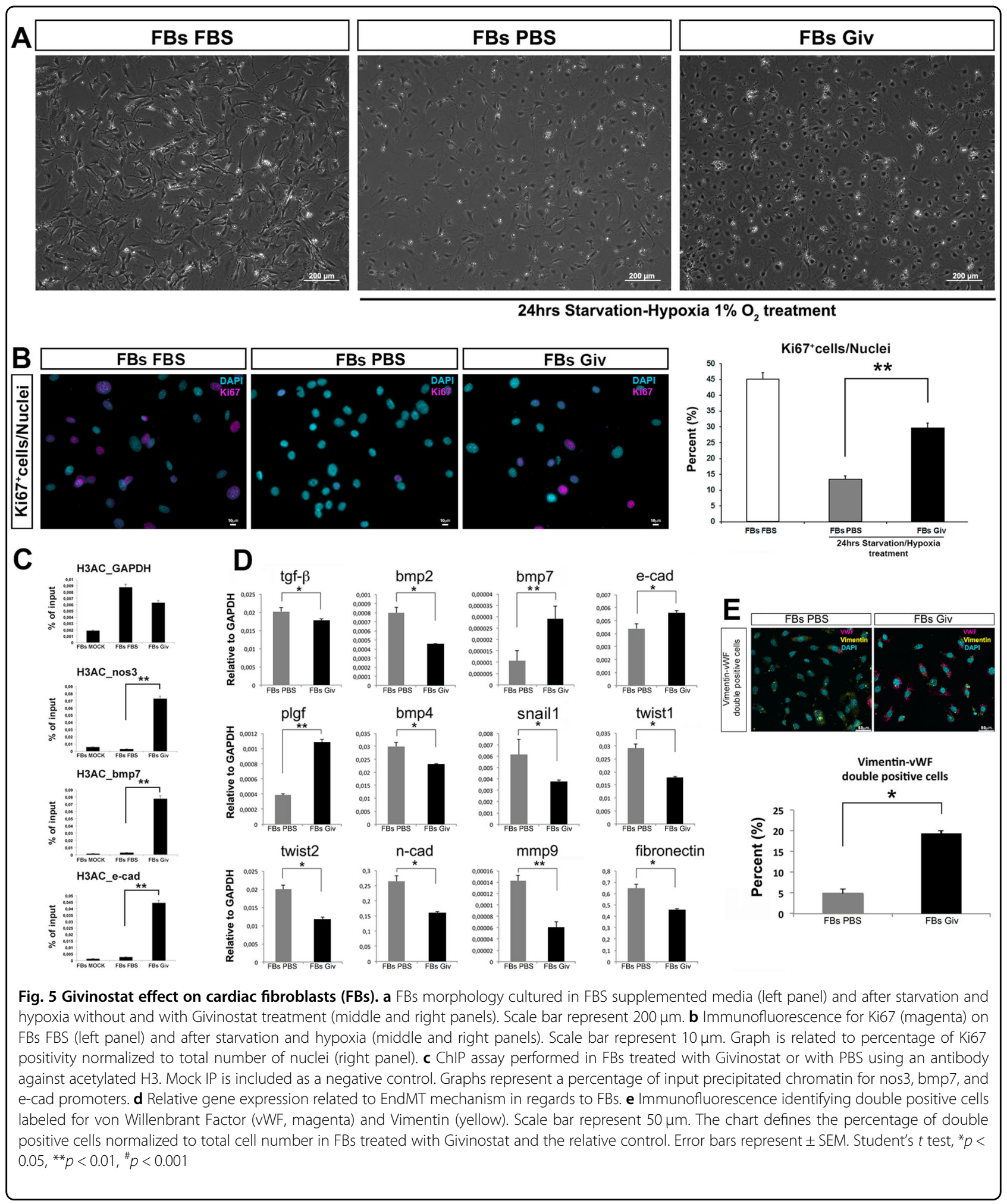

hypoxia, were collected after $24 \mathrm{~h}$. HUVECs culture media, after starvation, were replaced with those supernatant derived from FBs exposed to different experimental conditions. HUVECs were then exploited for angiogenesis detection test using a matrigel substrate. After $6 \mathrm{~h}$ we acquired the images of the cultures and analyzed by IMAGEJ integrated with Angiogenesis Software. Surprisingly, the number of knots, joints, and 


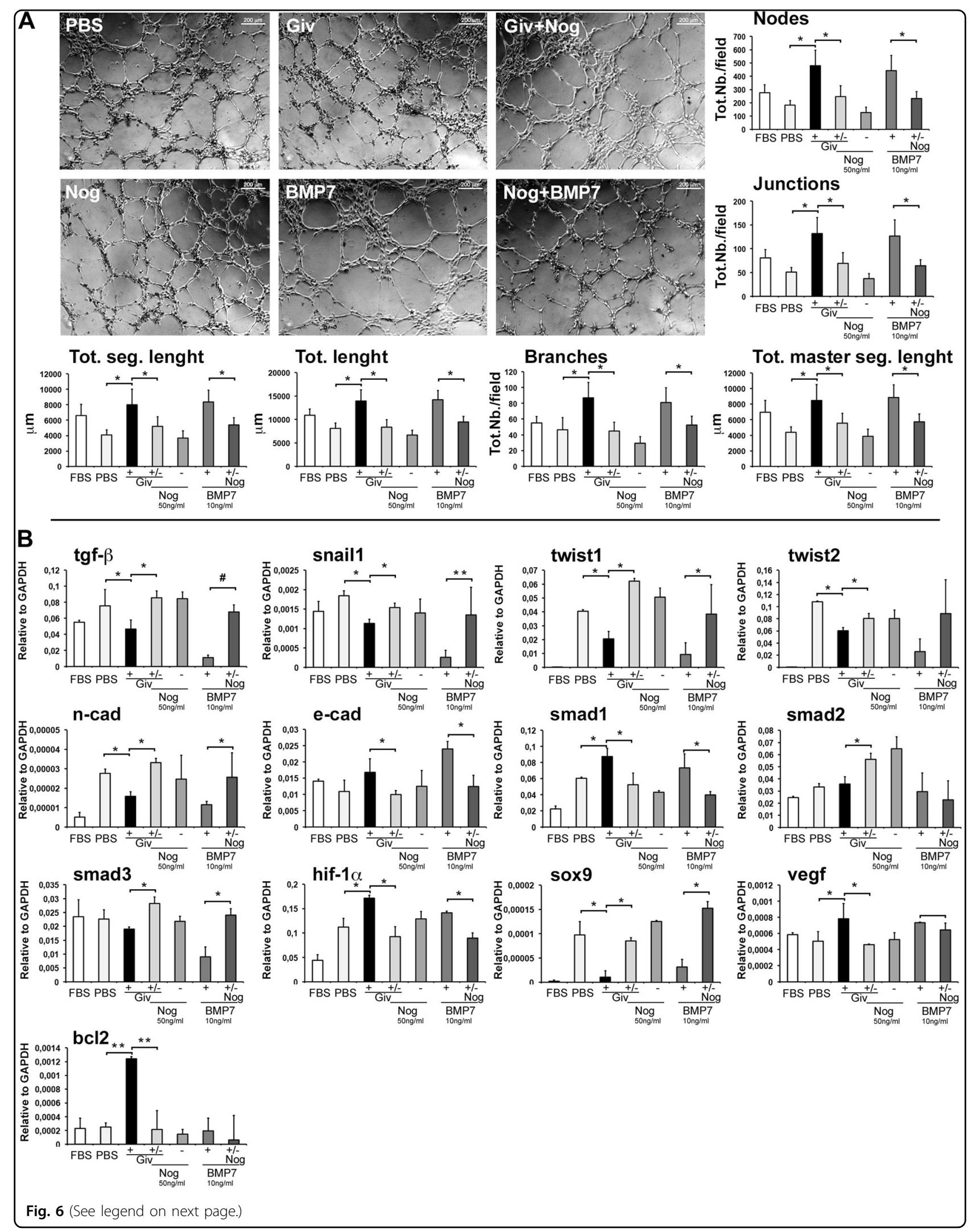


(see figure on previous page)

Fig. 6 Givinostat effect on HUVECs and perturbation studies. a Representative images of capillary-like structures formed by HUVECs pre-treated with Noggin or BMP7 and exposed to Givinostat/FBs-conditioned media or PBS after hypoxia. Diagrams quantifying nodes, junctions, total length segments, branches, total length and total segment length formed by HUVECs using ImageJ angiogenesis software. Scale bars represent $200 \mu \mathrm{mm}$. $\mathbf{b}$ Relative gene expression regarding EndMT mechanism (tgf- $\beta$, snail1, twist1/2, n-cad, e-cad, smad1/2/3, sox9) angiogenesis (hif- $1 a$ and vegf) and apoptosis (bcl2) in the different culture media. Error bars represent \pm SEM. Student's $t$ test, ${ }^{*} p<0.05,{ }^{*} p<0.01,{ }^{*} p<0.001$

branches, moreover the whole total length, that of the segments and that of the master segments, increased significantly in the group exposed to supernatant of FBs treated with Givinostat and in parallel with HUVECs given BMP-7 (Fig. 6a).

qRT-PCR experiments have shown that the expression of the genes related to the EndMT mechanism are significantly reduced (Fig. 6b). In addition, the supernatant of the FBs treated with Givinostat favored the expression of genes involved in angiogenesis (e-cad, smad1, vegf) (Fig. 6b), augmenting vessel formation by an auto-looping mechanism. Very surprising was the over-expression of genes such as bcl2 (Fig. 6b), which protects ECs against apoptosis for longer-lasting angiogenic performance; in fact, tgf- $\beta$ induces apoptotic cell death in HUVEC cultures down-regulating the expression of bcl- $2^{25}$. Additionally, the over-expression of hif- $1 \alpha$ (Fig. $6 \mathrm{~b}$ ) stimulates angiogenesis and increases blood flow ${ }^{26}$. Finally, sox9, a transcription factor that directly regulates the deposition of collagen type $2^{27}$ and the inhibition of proliferation, invasion, and EndMT in onco/plastic cells, is strongly downregulated in the treated group ${ }^{28}$. The results obtained from this study reveal that the beneficial effect of Givinostat is linked to the modulation of EndMT mechanism by promoting MET. On the other hand, its action is exercised in a multifactorial context, where every single cell population plays its role in contributing to the recovery of the damaged tissue.

\section{Discussion}

Our study strengthens the rationale regarding the role of HDACi in inducing sufficient epigenetic modifications to positively remodel the heart after an ischemic event. Although first generation of HDACi (TSA, SAHA) have been already demonstrated to possess this property reducing cardiac hypertrophy and fibrosis, the molecular mechanisms have not yet been elucidated. Givinostat, similarly to other HDACi, has anti-neoplastic activity on multiple myeloma and anti-inflammatory action on several autoimmune disorders ${ }^{29}$. In particular, Givinostat retains anti-inflammatory effect at low concentration as opposed to TSA ${ }^{30}$ and its efficacy was found to be $25-50$ fold more powerful than SAHA in vitro and in vivo ${ }^{31}$. However, the anti-fibrotic role of Givinostat in an in vivo model of heart failure has not yet been studied.
Our data highlight the role of Givinostat in preserving cardiac function following an acute cardiovascular insult. This phenomenon runs parallel to a decreased cardiac fibrosis that, together with cardiac tissue remodeling, observed earlier at day 3, exhibits premature features. This outcome is noteworthy, considering that both timing resolution and the arrest of further evolution of cardiac fibrosis after AMI represent the main key issues to positively address the healing response within the tissue ${ }^{32}$. To the best of our knowledge, only one study has reported the attenuation of fibrosis in presence of Givinostat, however in a model of corneal fibrosis ${ }^{33}$. Our results also show a reduced apoptosis in the whole cardiac muscle and an enhanced expression of $\mathrm{Cx} 43$, a known marker of functioning contractility ${ }^{34}$. The data suggests that Givinostat might act by decreasing the rate of cells loss, described to be exacerbated during infarct and negatively conditioning the compensatory re-adaptation of the cardiac muscle upon ischemic stress ${ }^{35}$ and in line with the ability of class I/II HDACi to control cardiac hypertrophy and foetal gene expression in $\mathrm{CMs}^{36}$. Interestingly, clinically approved pan HDCAi have been demonstrated able to induce cardiac-like reprogramming of stromal cells and cell death in cancer cells ${ }^{37}$. However, different pathophysiological disorders are capable to address diverse epigenetic assets. Thus, it is not surprising if contrasting effects are achieved by employing different HDACi.

Notably, HDACi can also target non-histone proteins therefore broadening their range of action. Besides, an enhanced vasculature ${ }^{38}$ was present, suggesting an additional ability of Givinostat in the adaptation of the vascular design upon cardiac hypoxia.

Our results also establish that the cardiac tissue responds to Givinostat treatment by altering its own epigenetic state. Accordingly, increased levels of acetylated Histone 3, a main relevant epigenetic modification associated with cardiovascular commitment, have been found. This correlates with the efficacy of Givinostat and is ascribable to modifications of the epigenetic state of the heart. Based on this premise, we also believe that Givinostat may exert a pleiotropic influence, resulting in an overall inhibition of the degeneration of the cardiac tissue truly due to its intrinsic deacetylation-based mechanism. Thus, the master regulators of the "maladaptive" stress response to AMI would be simply switched off or 
indirectly downregulated. Consequently, we investigated the gene profile and we found important cardiac tissue transcriptional and protein changes after treatment, resulting in a less fibrotic prone profile. This significant decreased in tgf- $\beta$ expression was observed at day 7 in vivo, in line with the maximal tgf- $\beta$ induced peak normally observed during $\mathrm{AMI}^{37}$. Although the trend for tgf- $\beta$ levels overlap with controls on long-term, it has been recently demonstrated that cardiac protective anti-fibrotic effects due to tgf- $\beta$ can be only achieved in the earliest phase of infarction ${ }^{35}$. Moreover, the decrease in tgf- $\beta$ mRNA levels is coherent with the modulation of markers associated to a potential reduction of the EndMT phenotype ${ }^{39}$. Notably, the efficacy of Givinostat cannot be uncoupled from the injury. In fact, in absence of AMI transcriptional changes in the tgf- $\beta / \mathrm{bmp}-7$ axis are not observed.

The EndMT triggered by ischemic events is one of the mechanisms that increase collagen secretion by inducing ECs to trans-differentiate into mesenchymal cells; recently it has also been shown a fundamental role of FBs in the same response, which consists in the trans-differentiation to myofibroblasts that can lead to an additional structure to the myocardium ${ }^{40}$. A second phase begins with an increase in fibrosis, which, once in excess, causes a stiffening of the heart wall such that systolic contraction becomes insufficient for correct blood ejection. The molecular mechanisms underlying these responses are driven by the expression of tgf- $\beta / \mathrm{bmp}-7$ axes. Indeed, increased expression of tgf- $\beta$ is capable of triggering the conversion of ECs into mesenchymal phenotype, while bmp-7 and p53 trigger the reverse process.

This study brought to light the relationship between the adjustment of this mechanism and the severity of the onset of heart failure. We have shown how Givinostat has the ability to improve heart performance by modulating the expression of tgf- $\beta$ at the cardiac FB level, through a protective action against apoptosis in muscle cells and by increasing angiogenesis. The improvement of postoperative conditions after HDACi treatment is attributable to a wide-range effect of Givinostat on the different cell populations. The secretion of angiogenic key factors, by FBs, after Givinostat administration ensures an increase in vascular formation that is essential for the regenerative phase in damaged organs. We cannot ignore that the inhibition of HDACs modulates the outcomes of whole cardiac system whose sum of effects produces a benefit. The broad action of Givinostat also involves populations such as MPs and, in general, inflammatory infiltrate, which modulates behavior and exert influence on cardiac populations. We believe that the wide-ranging effect of Givinostat is its best quality. The increased angiogenesis rather than the formation of collagen in the second phase of the compensatory response guarantee an ameliorative effect that could ensure an increased life expectancy.

In conclusion, our findings underline that cardioprotection can be achieved by employing Givinostat, therefore revealing a novel clinical applicability beyond DMD.

\section{Materials and methods}

\section{Animal model and surgery}

The study was conducted using female C57BL/6 wild type mice (10 weeks old). Acute Myocardial infarct (AMI) was performed through permanent ligation of the left descending coronary artery as already described ${ }^{41}$. Animals were anesthetized with intramuscular injection of ketamine $(10 \mathrm{mg} / \mathrm{ml})$ and xylazine $(1 \mathrm{mg} / \mathrm{ml})$ and divided into two groups: (1) AMI and daily saline-only injection and (2) AMI and daily intraperitoneal injections ${ }^{42,43}$ of Givinostat (10 $\mathrm{mg} / \mathrm{Kg} / \mathrm{d}$ dissolved in saline solution). Both saline and Givinostat have been administered after chest closure. Mice were handled and killed (cervical dislocation) in compliance with the European Convention on Animal Care. The experimental protocol was approved and numbered as Prot. N56/2014 Ministry of Health. They have also received human cares in accordance with the guidelines from Directive 2010/63/EU of the European Parliament on the protection of animals used for scientific purposes.

\section{Echocardiographic measurements}

Mice subjected to AMI were anesthetized by $2 \%$ isoflurane inhalation and imaged at day $0,1,3,7,15$, and 30 with VisualSonics Vevo $3100^{\circ}$ echocardiography. An M-mode scan of the left ventricle was assessed in the parasternal long axis view for measurements of intraventricular septal thickness, left ventricular posterior wall thickness, left ventricular dimension, and diastolic posterior wall velocity.

\section{Immunofluorescence}

Hearts were perfused through abdominal aortic cannulation and fixed in Neutral-buffered Formalin 10\%, dehydrated with ascending alcohols and finally included in paraffin to perform immunofluorescence on sections. Paraffin-embedded sections of 2- $\mu \mathrm{m}$ were prepared for histological analysis. The sections were dewaxed and rehydrated with descending alcohols and subjected to antigen retrieval procedure using citrate buffer $\mathrm{pH} 6.0$ before staining. Slides were washed with PBS and then incubated for $30 \mathrm{~min}$ at room temperature with a saturating solution consisting of $10 \%$ normal Donkey serum dissolved in PBS. After blocking the sections were incubated overnight at $4{ }^{\circ} \mathrm{C}$ with the following primary antibodies used at 1:100 dilution: mouse anti- $\alpha$ sarcomeric actin ( $\alpha$-Sarc, Genetex GTX29465), anti-smooth muscle actin ( $\alpha$-SMA, SIGMA A2547), Connexin 43 (Cx43, Abcam, Ab3512). For Cx43 immunofluorescence images 
see "Method for the measurement of Connexin expression" and Supplementary Figure 5. Apoptosis was assessed by TUNEL assay (ApoAlert ${ }^{\mathrm{TM}}$ DNA Fragmentation Assay Kit, Clontech, Cat. N.630107) according to the manufacturer's protocol. After washing with PBS, slides were incubated for $1 \mathrm{~h}$ with fluorescent-conjugated secondary antibodies (1:500 dilution). Negative controls were tested by incubation of only the secondary antibody without primary antibody incubation. All secondary antibodies were negative for non-specific staining. After three washes, sections were mounted with Vectashield mounting medium (VECTOR, H-1200) with DAPI.

Images were acquired by Confocal microscope TCS SP5 (Leica Microsystem). Analysis was performed in sequential scanning mode to rule out cross bleeding between channels.

Cells were fixed in 4\% PFA and processed for immunofluorescence assay. Briefly, after fixation with $4 \%$ PFA for $10 \mathrm{~min}$, cells were incubated overnight at $4{ }^{\circ} \mathrm{C}$ with the following primary antibodies used at 1:100 dilution: antiKi67 (Ki67, Abcam, 15580) anti-Vimentin (Vimentin, Abcam, 45939), anti-Von Willebrand Factor (vWF, Abcam 11713). Cells were then incubated with the appropriate secondary fluorophore-conjugated antibody. Nuclei were stained with DAPI. Images were acquired by Zeiss Microscope associated with a Nikon camera (Axio Observer A1, Zeiss, Germany).

\section{Method for the measurement of connexin expression}

Immunofluorescence for $\mathrm{Cx} 43$ was converted into black and white using an image processing program ImageJ, according to the following sequence: Process $>$ Binary $>$ Convert to Binary. Connexin 43 quantification, area of particles, was obtained using the command Analyze>Analyze Particles (Supplementary Figure 5).

\section{Scar size assessment and collagen assay}

Paraffin-embedded sections $(2-\mu \mathrm{m})$ were dewaxed and rehydrated with descending alcohols and stained with Masson's Trichrome KIT (Sigma-Aldrich, No.HT15, St. Louis, MO, USA) according to the manufacturer's protocol. Scar size (percentage) was expressed as ratio of fibrotic area (stained in blue) on total area, using an image processing program ImageJ.

\section{Real time PCR}

The evaluation of genes expression was performed by quantitative Real-time PCR on a 7900HT Fast Real-time PCR System equipped with SDS software (Applied Biosystems). The expression data were normalized using the Ct values of GAPDH as the housekeeping gene. Tissue samples were homogenized with Tissue Ruptor in $1 \mathrm{ml}$ of TRIZOL reagent (Invitrogen, Life Technologies, 15596026). Samples homogenized were incubate for 5 min at $15^{\circ} \mathrm{C}$ to $30^{\circ} \mathrm{C}$ to permit a complete dissociation of nucleoprotein complexes and $0.2 \mathrm{ml}$ of chloroform were added per $1 \mathrm{ml}$ of TRIZOL. Tubes were vigorously shacked for $15 \mathrm{~s}$ and incubated at $15-30{ }^{\circ} \mathrm{C}$ to $3 \mathrm{~min}$ and centrifuged at $12,000 \times g$ for $15 \mathrm{~min}$ at $4{ }^{\circ} \mathrm{C}$. Following centrifugation, the mixture separates into three phases and RNA remains exclusively in the upper aqueous phase, the volume was about $60 \%$ of the TRIZOL volume used. The aqueous phase was transferred to a fresh tube and RNA was precipitated with $0.5 \mathrm{ml}$ of isopropyl alcohol. Samples were incubated at $15-30^{\circ} \mathrm{C}$ to $10 \mathrm{~min}$ and centrifuged $12,000 \times g$ for $10 \mathrm{~min}$ at $4^{\circ} \mathrm{C}$. After centrifuge, supernatant was removed and RNA was visible as a pellet bottom of tube. Successively pellet was washed with $1 \mathrm{ml}$ of $75 \%$ ethanol and centrifuged $7,500 \times g$ for $5 \mathrm{~min}$ at $4{ }^{\circ} \mathrm{C}$. At the end of procedure RNA pellet was air-dried and dissolved in $30 \mu \mathrm{l}$ of RNasi free water. RNA quantity was determined by measuring absorbance at $260 \mathrm{~nm}$ using a NanoDrop UV-VIS spectrophotometer. TaqMan Fast Universal PCR Master Mix (Superscript VILO Invitrogen 11754-050) was employed to reverse transcribe RNA into single-stranded cDNA. The evaluation of genes expression was performed by quantitative real-time PCR with SYBER Green PCR Master Mix 4309155 (Applied Biosystems) on a 7900HT Fast Real-time PCR System equipped with SDS software (Applied Biosystems). Quantitative PCR parameters for cycling were set up as follows: $50^{\circ} \mathrm{C}$ incubation for $2 \mathrm{~min}, 95^{\circ} \mathrm{C}$ for $10 \mathrm{~min}, 40$ cycles of PCR at $95^{\circ} \mathrm{C}$ for $15 \mathrm{~s}$, and $60^{\circ} \mathrm{C}$ for $1 \mathrm{~min}$ (see Table 1 for primer sequences). All reactions were performed in a $15 \mu \mathrm{l}$ reaction volume in triplicate. The expression data were normalized using the $\mathrm{Ct}$ values of GAPDH as the housekeeping gene.

\section{Western blotting}

Protein samples were prepared from left ventricle and immunocomplexes were detected by chemiluminescent reaction followed by densitometry analyses with the software ImageJ. GAPDH expression levels were used to normalize the results. Tissue samples were homogenized in $500 \mu \mathrm{l}$ of lysis buffer $(50 \mathrm{mM}$ TRIS $\mathrm{HCl} \mathrm{pH} 7.5,0.6 \mathrm{M}$ sucrose, $50 \%$ glycerol, $1 \%$ TRITON, $50 \mathrm{Mm} \mathrm{NaCl}, 10 \mathrm{mM}$ $\mathrm{NaF}, 2 \mathrm{mM}$ NaOV4, $1 \mathrm{mM}$ PMSF, $5 \mathrm{mM} \beta$-glycerolphosphate, $1000 \times$ protease inhibitors) using the Tissue Ruptor disposable (4 times for $5-10 \mathrm{~s}$ in ice). Then, lysates were sonicated $5 \mathrm{~s}$ for 4 times, incubated for $20-30 \mathrm{~min}$ at $4{ }^{\circ} \mathrm{C}$ and centrifuged $12,000 \times g$ for $15 \mathrm{~min}$ at $4{ }^{\circ} \mathrm{C}$. Protein concentration was determined measuring absorbance at $598 \mathrm{~nm}$ using a spectrophotometer. For western blot analysis proteins were resolved by SDS-polyacrylamide gel electrophoresis and transferred to nitrocellulose membranes $(0.45 \mu \mathrm{m}$ pore size, BIO-RAD). Blots were blocked for $45 \mathrm{~min}$ in $5 \%$ non-fat dry milk (Sigma-Aldrich). The membranes were then incubated overnight at $4{ }^{\circ} \mathrm{C}$ with the following primary antibodies: Histone 3 (1:3000, 
Abcam, Ab10799) or Acetyl Histone 3 (1:3000, Merck Millipore, Cat. N.07-352), Cx43 (1:500, Cell Signaling, \#3512), TGF- $\beta$ (1:200, Abcam 66043). Binding of the primary antibody was detected with the use of peroxidaseconjugated secondary antibodies (1:5000, $\alpha$-Rabbit NA934AV, $1: 10,000 \alpha$-Mouse LNXa931/AE both GE Healthcare Life Sciences) for $1 \mathrm{~h}$ at room temperature. Immunocomplexes were detected by chemiluminescent reaction (ECL kit; Merck Millipore) followed by densitometry analyses with the software ImageJ. GAPDH expression levels were used to normalize the results.

\section{Angiogenesis assays}

The effects of Givinostat-exposed fibroblasts secretomics on endothelial cell ability to form capillary-like structures on basement membrane matrix, was assessed in vitro, by morphogenesis assay. Human fibroblasts were starved for $6 \mathrm{~h}$, then exposed to oxygen hypoxia $1 \%$ and subdivided in experimental groups for perturbation studies and treated with: $50 \mathrm{nM}$ of Givinostat, $50 \mathrm{nM}$ of Givinostat and $50 \mathrm{ng} / \mathrm{ml}$ of Noggin, $50 \mathrm{ng} / \mathrm{ml}$ of Noggin, $10 \mathrm{ng} / \mathrm{ml}$ of BMP7, $50 \mathrm{ng} / \mathrm{ml}$ of Noggin and $10 \mathrm{ng} / \mathrm{ml}$ of BMP7 for $18 \mathrm{~h}$, while the control group was treated with PBS. After $24 \mathrm{~h}$ supernatants were collected.

HUVECs were grown on EBM2 complete medium and when $80 \%$ confluent and exposed to fibroblasts secretomics. Positive and negative controls received 10\% FBS or serum free EBM2 medium, respectively. A 96-well plate, pre-chilled at $-20^{\circ} \mathrm{C}$, was carefully filled with $75 \mu \mathrm{l}$ of liquid matrigel (BD Biosciences, Milan, Italy) per well at $4{ }^{\circ} \mathrm{C}$ and let polymerized for $1 \mathrm{~h}$ at $37^{\circ} \mathrm{C}$. $1.5 \times 10^{3}$ HUVEC cells/well were suspended in $100 \mu \mathrm{l}$ of EBM2 medium containing $100 \mathrm{ng} / \mathrm{ml}$ VEGF and $100 \mathrm{ng} / \mathrm{ml} \mathrm{FGF2}$ alone, or with the conditioned medium and layered on the top of the polymerized matrigel. The effects on HUVECs tube formation were captured after $6 \mathrm{~h}$ incubation using a Zeiss Microscope associated with a Nikon camera (Axio Observer A1, Zeiss, Germany) and were quantified using ImageJ software and the "Angiogenesis Analyzer" tool.

\section{Bone marrow-derived macrophages}

Bone marrow cells were isolated from femur and tibia of C57 mice. One million bone marrow cells were plated in 10 $\mathrm{cm}$ plates in $5 \mathrm{ml}$ of BM-medium (DMEM supplemented with $20 \%$ low-endotoxin fetal bovine serum, 30\% L929-cell conditioned medium, $1 \%$ L-glutamine, $1 \%$ Pen/Strep, 0.5\% Na Pyruvate, $0.1 \% \beta$-mercaptoethanol) and fed with $2.5 \mathrm{ml}$ of fresh medium every 2 days. After 6 days cells were semiconfluent (80\%) and used for co-culture experiments.

\section{Neonatal cardiomyocytes isolation}

Hearts were isolated from 40 decapitated 1-to 3-day-old neonatal wild type mice with the atria dissected away were minced and digested with $108 \mathrm{U} / \mathrm{ml}$ collagenase type II
(Worthington) and $0.9 \mathrm{mg} / \mathrm{ml}$ pancreatin (Life Technologies, Grand Island, NY) to obtain free cells. Myocytes were plated on gelatin-coated dishes overnight in DMEM/ medium 199 (4:1) supplemented with 10\% horse serum, $5 \%$ fetal calf serum, $2 \mathrm{mM}$ L-glutamine (Gibco), $100 \mathrm{U} / \mathrm{ml}$ penicillin, and $100 \mathrm{mg} / \mathrm{ml}$ streptomycin (EuroClone) at a density of $1 \times 10^{5}$ cells $/ \mathrm{cm}^{2}$. The next day cells were rinsed three times and the plating medium was replaced with serum-free medium consisting only of DMEM/ medium 199 (4:1), $2 \mu \mathrm{M}$ L-glutamine (Gibco), $100 \mathrm{U} / \mathrm{ml}$ penicillin, and $100 \mathrm{mg} / \mathrm{ml}$ streptomycin (EuroClone); 10 $\mu \mathrm{M}$ cytosine- $\beta$ - $\mathrm{d}$-furanoarabinoside was added to stop proliferation of non-cardiomyocytes and cultures contained $>95 \%$ cardiac myocytes ${ }^{44}$. Cells were serum starved for $6 \mathrm{~h}$ before starting the experiments of co-culture with macrophages.

\section{Human fibroblasts}

Stable lines of cryopreserved human cardiac fibroblasts are available in our laboratory.

\section{Mouse fibroblasts isolation}

Hearts were isolated from 10 adult C57 mice and digested with $0.1 \mathrm{mg} / \mathrm{ml}$ of collagenase type2, $337 \mathrm{U} / \mathrm{mg}$ (Worthington) dissolved in DMEM (Gibco) at $37^{\circ} \mathrm{C}$ in a shaking water bath for $10 \mathrm{~min}$. The supernatant, containing free cells, was then collected and kept on ice. The digestion step was repeated three times. Cell suspensions from each digestion were pooled, filtered through a $70 \mu \mathrm{m}$ strainer (Falcon), and centrifuged at 1200 r.p.m. for $5 \mathrm{~min}$.

The cell pellet was then resuspend in a fibroblast medium (FB medium) containing high glucose DMEM (Gibco) supplemented with 10\% fetal bovine serum (FBS, Invitrogen), $2 \mathrm{mM}$ L-glutamine (Gibco), $100 \mu \mathrm{M}$ NEAA (Gibco), $100 \mathrm{U} / \mathrm{ml}$ penicillin, and $100 \mathrm{mg} / \mathrm{ml}$ streptomycin (EuroClone). Cells obtained from individual animals were plated in a singular $100 \mathrm{~mm}$ plate in $10 \mathrm{ml}$ of $\mathrm{FB}$ medium and incubated at $37^{\circ} \mathrm{C}$ and $5 \% \mathrm{CO}_{2}$.

\section{Co-culture experiments}

Macrophages were plated alone or in co-culture with neonatal cardiomyocytes or cardiac fibroblasts (3:1) using a transwell system and exposed to $50 \mathrm{nM}$ of Givinostat. After $1 \mathrm{~h}$ macrophages were polarized toward M1 phenotype with $10 \mathrm{ng} / \mathrm{ml}$ of lipopolysaccharide (LPS) or to M2 phenotype with $10 \mathrm{ng} / \mathrm{ml}$ of interleukin (IL-4), M0 macrophages were plated without polarization factors. Cells were collected in $1 \mathrm{ml}$ of TRIZOL reagent after $24 \mathrm{~h}$ of treatment.

\section{Chromatin immunoprecipitation (ChIP)}

Cardiac fibroblasts treated with Givinostat and the relative control, were fixed by adding directly to the culture medium formaldehyde 36.5\% (Sigma Aldrich) to a final concentration of $1 \%$ and incubated for $10 \mathrm{~min}$ at 
Table 2 Mouse and human primer sequences for quantitative RT-PCR analysis

\begin{tabular}{|c|c|c|c|}
\hline Gene symbol & RefSeq Acc.number & Sense-forward primer & Antisense-reverse primer \\
\hline \multicolumn{4}{|l|}{ MOUSE } \\
\hline anf & NM_008725.2 & TCTTCTTCGTCTTGGCCTIT & GACCTCATCTTCTACCGGCA \\
\hline $\mathrm{bcl} 2$ & NM_009741 & GAGTACCTGAACCGGCATCT & TTGTTTGGGGCAGGTTTGTC \\
\hline bmp2 & NM_007553 & GAAGTTCCTCCACGGCTTCT & AGATCTGTACCGCAGGCACT \\
\hline bmp4 & NM_007554 & AGCCAACACTGTGAGGAGTT & GGATGCTGCTGAGGTTGAAG \\
\hline bmp7 & NM_007557 & GTGGTATCGAGGGTGGAAGA & ACAAGGCCGTCTTCAGTACC \\
\hline brachiury & NM_009309 & GGTGCTGAAGGTAAATGTGTC & GGCTGTAATCTCTCCTCATTCTGC \\
\hline col1a1 & NM_007742.3 & CCTCAGGGTATTGCTGGACA & GAAGGACCTTGTTTGCCAGG \\
\hline col1a2 & NM_007743.2 & GGAACAAATGGGCTCACTGG & CAAGTCCTCTGGCACCTGTA \\
\hline col3a1 & NM_009930.2 & CCCAACCCAGAGATCCCATT & GGTCACCATTTCTCCCAGGA \\
\hline e-cadherin & NM_009864.2 & CTGGACCGAGAGAGTTACCC & GTGCTTGGGTTGAAGACAGG \\
\hline $\mathrm{f} 4 / 80$ & NM_010130.4 & CAACCTGCCACAACACTCTC & ATGAGCAGCTGTAGGATCCC \\
\hline gapdh & NM_001289726 & CACCATCTCCCAGGAGCGCAG & СCTTCTCCATGGTGGTGCAGAC \\
\hline gata4 & NM_001310610.1 & GGAAGACACCTCAATCTCGT & CACAGGCATTGTACAGGTAG \\
\hline $\mathrm{il}-1 \mathrm{a}$ & NM_010554.4 & ATGTATGCCTACTCGTCGGG & CAACTCCTTCAGCAACACGG \\
\hline $\mathrm{il}-1 \beta$ & NM_008361.4 & TGACGGACCCCAAAAGATGA & TCTCCACAGCCACAATGAGT \\
\hline il-4 & NM_021283 & CGAGCTCACTCTCTGTGGTG & TGAACGAGGTCACAGGAGAA \\
\hline mef2 & NM_001170537.1 & TCAGTTGGGAGTTTGCACTA & TGGTGGTACGGTCTCTAGGA \\
\hline mmp9 & NM_013599.4 & AAAACCTCCAACCTCACGGA & GTGGTGTTCGAATGGTCTTT \\
\hline mhc-a & NM_001164171.1 & CCAACACCAACCTGTCCAAG & CTCGTCGTGCATCTTCTTGG \\
\hline$m h c-\beta$ & NM_080728.2 & CCTGGAGAATGACAAGCAGC & GAGCTTCTTCTGCAGCTGAC \\
\hline n-cadherin & NM_007664.4 & TGGCTGAAAATAGACCCCGT & TTCCTGTCCCACTCATAGGC \\
\hline $\mathrm{nk} \times 2.5$ & NM_008700 & CAGTGGAGCTGGACAAAGCT & TAGCGACGGTTCTGGAATCA \\
\hline p53 & NM_011640 & ACAGTCGGATATCAGCCTCG & GCTTCACTCGGGTCTTCAAA \\
\hline plgf & NM_008827 & GTTGGCTGTGCATTCCCAG & TACACCAGCTTCTCCATGGG \\
\hline sma & NM_007392.3 & CCTCTGGACGTACAACTGGT & GGTAGTCGGTGAGATCTCGG \\
\hline snail1 & NM_011427.2 & CGACTACCTAGGTCGCTCTG & CTGCTGGAAGGTGAACTCCA \\
\hline snail2 & NM_011415.2 & CGAACTGGACACACACACAG & AAAGGAGAGTGGAGTGGAGC \\
\hline tnf-a & NM_013693.3 & CGTCGTAGCAAACCACCAAG & GGCAGAGAGGAGGTTGACTT \\
\hline tnni3 & NM_009406.4 & AGCAGGTGAAGAAGGAGGAC & GCATCGATATTCTTGCGCCA \\
\hline twist1 & NM_011658.2 & GCCAGGTACATCGACTTCCT & CCAGACGGAGAAGGCGTAG \\
\hline twist2 & NM_007855.3 & AAGATCATCCCCACGCTCC & ATTGTCCATCTCGTCGCTCT \\
\hline $\operatorname{tg}-\beta 1$ & NM_011577 & CAACCCAGGTCCTTCCTAAA & GGAGAGCCCTGGATACCAAC \\
\hline casp3 & NM_009810.3 & GAGCAGCTITGTGTGTGTGTGA & TTCGGCTTTCCAGTCAGACT \\
\hline \multicolumn{4}{|l|}{ HUMAN } \\
\hline $\mathrm{bcl} 2$ & NM_000633.2 & GCCCTGTGGATGACTGAGTA & GAAATCAAACAGAGGCCGCA \\
\hline e-cadherin & NM_004360.3 & ACAACAAGCCCGAATTCACC & GGTGTTCACATCATCGTCCG \\
\hline fibronectin & NM_212482.2 & CCCCATTCCAGGACACTTCT & AGGGTTCTTCATCAGTGCCA \\
\hline hif-1a & NM_001530.3 & ATITGGCAGCAACGACACA & GGGTGAGGGGAGCATTACAT \\
\hline
\end{tabular}


Table 2 continued

\begin{tabular}{|c|c|c|c|}
\hline Gene symbol & RefSeq Acc.number & Sense-forward primer & Antisense-reverse primer \\
\hline sma & NM_001141945.1 & CTGCTGAGCGTGAGATTGTC & TCAAGGGAGGATGAGGATGC \\
\hline mmp9 & NM_004994.2 & CGCTACCACCTCGAACTITG & ATAGGGTACATGAGCGCCTC \\
\hline n-cadherin & NM_001792.4 & AGGGATCAAAGCCTGGAACA & TTGGAGCCTGAGACACGATT \\
\hline smad1 & NM_005900.2 & GTACTTCCTCCTGTGCTGGT & TGGAAAAGTGGCGTTGAGTG \\
\hline smad2 & NM_005901.5 & GACACCAGTITTGCCTCCAG & СTCTGTGGCTCAATTCCTGC \\
\hline smad3 & NM_001145104.1 & CTAGGGCTGCTCTCCAATGT & AAGACCTCCCCTCCGATGTA \\
\hline snail1 & NM_005985.3 & AAGCCTAACTACAGCGAGCT & GAGTCCCAGATGAGCATTGG \\
\hline snail2 & NM_003068.4 & AGCATTTCAACGCCTCCAAA & TGGTTGTGGTATGACAGGCA \\
\hline sox9 & NM_000346.3 & GGCAAGCTCTGGAGACTTCT & CGCGGCTGGTACTTGTAATC \\
\hline $\operatorname{tgf}-\beta 1$ & NM_000660.5 & CAGCAGGGATAACACACTGC & CATGAGAAGCAGGAAAGGCC \\
\hline twist1 & NM_000474.3 & AGTCTTACGAGGAGCTGCAG & ATCTTGCTCAGCTTGTCCGA \\
\hline twist2 & NM_057179.2 & AGAGCGACGAGATGGACAAT & CTAGTGGGAGGCGGACATG \\
\hline vegf & NM_001171624.1 & TCTACCTCCACCATGCCAAG & TGATGATTCTGCCCTCCTCC \\
\hline casp3 & NM_032991.2 & AAAATACCAGTGGAGGCCGA & GCACAAAGCGACTGGATGAA \\
\hline \multicolumn{4}{|l|}{ ChIP_sequences } \\
\hline bmp7 & NM_007557.3 & GATGAGCCAGGTCCAAGAGT & AGCAACTAAGGGCTGTGCTA \\
\hline e-cadherin & NM_009864.3 & ACACGGAGGGAGAACAATGT & CCCCAAGTAGCAGCATCCTA \\
\hline nos3 & NM_008713.4 & TAGGAGAGGAGCAAGGGTGA & TACAGTGGGAGGGCTTCGAG \\
\hline
\end{tabular}

room temperature. Then, Tris- $\mathrm{HCl} \mathrm{pH} 7.6$ was added to a final concentration of $125 \mathrm{mM}$ to quench formaldehyde and cells were washed with cold PBS for $10 \mathrm{~min}$ on ice and rapidly collected, centrifuged at $400 \times g$ for $5 \mathrm{~min}$ at $4{ }^{\circ} \mathrm{C}$. Cells were lysated for $10 \mathrm{~min}$ in $\mathrm{L} 1$ buffer $(50 \mathrm{mM}$ Hepes-KOH pH7.5, $140 \mathrm{mM} \mathrm{NaCl}, 1 \mathrm{mM}$ EDTA, 10\% glycerol, $0.5 \%$ NP-40, $0.25 \%$ Triton X-100 supplemented with protease inhibitors) and centrifuged at $400 \times g$ for 5 min at $4{ }^{\circ} \mathrm{C}$. Cell pellets were resuspended gently in $\mathrm{L} 2$ buffer $(10 \mathrm{mM}$ Tris- $\mathrm{HCl}$ pH8.0, $200 \mathrm{mM} \mathrm{NaCl}, 1 \mathrm{mM}$ EDTA, $0.5 \mathrm{mM}$ EGTA) for $10 \mathrm{~min}$ on the wheel. After, nuclei were pelleted at $400 \times g$ for $5 \mathrm{~min}$ at $4{ }^{\circ} \mathrm{C}$ and resuspended in L3 buffer $(10 \mathrm{mM}$ Tris- $\mathrm{HCl} \mathrm{pH} 8.0,100$ $\mathrm{mM} \mathrm{NaCl}, 1 \mathrm{mM}$ EDTA, $0.5 \mathrm{mM}$ EGTA, $0.1 \%$ sodium dodecyl sulfate, $0.5 \% \mathrm{~N}$-lauroylsarcosine). Chromatin was sheared by sonication (maximum power $30 \mathrm{~s}$ ON, $1 \mathrm{~min}$ OFF, 5 min for 3 times). Chromatin IP was performed overnight on the wheel with $5 \mu \mathrm{g}$ of Acetyl Histone 3 (Merck Millipore, Cat. N.07-352). Immunoprecipitated samples were washed six times (5 min each) with wash buffer $(50 \mathrm{mM}$ Hepes- $\mathrm{KOH} \mathrm{pH}$ 7.6, $500 \mathrm{mM} \mathrm{LiCl}, 1 \mathrm{mM}$ EDTA, 1\% NP-40, 0.7\% Na-Deoxycholate) followed by a washing in TE1X with $50 \mathrm{mM} \mathrm{NaCl}$. Cross-linking was reversed at $65^{\circ} \mathrm{C}$ overnight in elution buffer $(10 \mathrm{mM}$ Tris- $\mathrm{HCl}$ pH 8.0, 5 mM EDTA, $300 \mathrm{mM} \mathrm{NaCl}, 0.5 \%$ SDS), and DNA was extracted from beads by standard phenol/ chloroform extraction, precipitated, and resuspended in $50 \mu \mathrm{l}$ of $\mathrm{H}_{2} \mathrm{O}$. About one-twentieth of the immunoprecipitated DNA was used in each PCR.

Quantitative real-time PCR reactions were performed in duplicate (precipitated DNA samples as well as serially diluted input DNA) with SYBER Green PCR Master Mix 4309155 (Applied Biosystems) on a 7900HT Fast Realtime PCR System equipped with SDS software (Applied Biosystems). Relative enrichment was calculated as ChIP/ input ratio. Primer sequences in Table 2.

\section{Statistical analysis}

Statistical analysis was carried out using GraphPad (Software Inc., La Jolla, CA, USA). Values presented are mean \pm SEM. Differences between sample means at each time point were evaluated with Student's $t$-test. $P$-value of $<0.05$ was considered statistically significant. $P$ values for each experiment are shown in supplementary information.

\section{Acknowledgements}

We thank Italfarmaco for supply of Givinostat (ITF2357). We also acknowledge Fondazione Roma for the VisualSonics Vevo $3100^{\circledR}$.

\section{Author details}

${ }^{1}$ Institute of Cell Biology and Neurobiology (IBCN), National Research Council of Italy (CNR), Monterotondo Scalo, Rome 00015, Italy. ${ }^{2}$ Operational Research Unit, Fondazione di Ricerca e Cura Giovanni Paolo II, Largo Gemelli 1,

Campobasso, Italy. ${ }^{3}$ RCCS Fondazione Santa Lucia, Rome 00142, Italy. ${ }^{4}$ Centro 
di Riferimento per la Medicina di Genere Istituto Superiore di Sanità Viale Regina Elena, 299 Roma, Italy. ${ }^{5}$ Department of Medical Surgical Sciences and Biotechnologies, Sapienza University of Rome, 04100 Latina, Italy. ${ }^{6}$ Department of AngioCardioNeurology, IRCCS NeuroMed, 86077 Pozzilli (IS), Italy. ${ }^{7}$ Development, Aging and Regeneration Program, Sanford Burnham Prebys Medical Discovery Institute, La Jolla, CA 92037, USA

\section{Conflict of interest}

The authors declare that they have no conflict of interest.

\section{Publisher's note}

Springer Nature remains neutral with regard to jurisdictional claims in published maps and institutional affiliations.

Supplementary information The online version of this article (https://doi. org/10.1038/s41419-017-0174-5) contains supplementary material.

Received: 29 September 2017 Revised: 10 November 2017 Accepted: 13 November 2017

Published online: 25 January 2018

\section{References}

1. Gupta, A. et al. Predictors of ischemic stroke in rheumatic heart disease. $J$ Stroke Cerebrovasc. Dis. 24, 2810-2815 (2015).

2. Usunier, B., Benderitter, M., Tamarat, R. \& Chapel, A. Management of fibrosis: the mesenchymal stromal cells breakthrough. Stem Cells Int. 2014, 340257 (2014).

3. Kumar, V. \& Gill, K. D. Oxidative stress and mitochondrial dysfunction in aluminium neurotoxicity and its amelioration: a review. Neurotoxicology 41 154-166 (2014)

4. Epelman, S., Liu, P. P. \& Mann, D. L. Role of innate and adaptive immune mechanisms in cardiac injury and repair. Nat Rev. Immunol. 15, 117-129 (2015).

5. Siciliano, C. et al. Cardiosphere conditioned media influence the plasticity of human mediastinal adipose tissue-derived mesenchymal stem cells. Cell Transplant. 24, 2307-2322 (2015).

6. Wang, Y. et al. Dysregulation of histone acetyltransferases and deacetylases in cardiovascular diseases. Oxid. Med. Cell Longev. 2014, 641979 (2014).

7. Yang, J. Y., Wang, Q., Wang, W. \& Zeng, L. F. Histone deacetylases and cardiovascular cell lineage commitment. World J. Stem Cells 7, 852-858 (2015).

8. Cao, D. J. et al. Histone deacetylase (HDAC) inhibitors attenuate cardiac hypertrophy by suppressing autophagy. Proc. Natl Acad. Sci. USA 108 4123-4128 (2011).

9. Liao, C. H. et al. Cardiac mast cells cause atrial fibrillation through PDGF-Amediated fibrosis in pressure-overloaded mouse hearts. J. Clin. Invest. 120 242-253 (2010).

10. McKinsey, T. A. Therapeutic potential for HDAC inhibitors in the heart. Annu. Rev. Pharmacol. Toxicol. 52, 303-319 (2012).

11. Singh, N. et al. Histone deacetylase 3 regulates smooth muscle differentiation in neural crest cells and development of the cardiac outflow tract. Circ. Res. 109, 1240-1249 (2011)

12. Backs, J., Song, K., Bezprozvannaya, S., Chang, S. \& Olson, E. N. CaM kinase selectively signals to histone deacetylase 4 during cardiomyocyte hypertrophy. J. Clin. Invest. 116, 1853-1864 (2006).

13. Leoni, F. et al. The histone deacetylase inhibitor ITF2357 reduces production of pro-inflammatory cytokines in vitro and systemic inflammation in vivo. Mol. Med. 11, 1-15 (2005)

14. Passamonti, F., Maffioli, M. \& Caramazza, D. New generation small-molecule inhibitors in myeloproliferative neoplasms. Curr. Opin. Hematol. 19, 117-123 (2012).

15. Shein, N. A. \& Shohami, E. Histone deacetylase inhibitors as therapeutic agents for acute central nervous system injuries. Mol. Med. 17, 448-456 (2011).

16. Vojinovic, J. et al. Safety and efficacy of an oral histone deacetylase inhibitor in systemic-onset juvenile idiopathic arthritis. Arthritis Rheum. 63, 1452-1458 (2011).

17. Felice, C., Lewis, A., Armuzzi, A., Lindsay, J. O. \& Silver, A. Review article: selective histone deacetylase isoforms as potential therapeutic targets in inflammatory bowel diseases. Aliment. Pharmacol. Ther. 41, 26-38 (2015).

18. Consalvi, S., Saccone, V. \& Mozzetta, C. Histone deacetylase inhibitors: a potential epigenetic treatment for Duchenne muscular dystrophy. Epigenomics 6, 547-560 (2014).
19. Duangkumpha, K. et al. BMP-7 blocks the effects of TGF-beta-induced EMT in cholangiocarcinoma. Tumour Biol. 35, 9667-9676 (2014)

20. Zeisberg, M. et al. BMP-7 counteracts TGF-beta1-induced epithelial-tomesenchymal transition and reverses chronic renal injury. Nat. Med. 9, 964-968 (2003).

21. Gogiraju, R. et al. Endothelial p53 deletion improves angiogenesis and prevents cardiac fibrosis and heart failure induced by pressure overload in mice. $J$. Am. Heart Assoc. 4, pii: e001770 (2015).

22. Chen, $X$. et al. Requirement for the histone deacetylase Hdac3 for the inflammatory gene expression program in macrophages. Proc. Natl Acad. Sci. USA 109, E2865-E2874 (2012).

23. Prabhu, S. D. \& Frangogiannis, N. G. The biological basis for cardiac repair after myocardial infarction: from inflammation to fibrosis. Circ. Res. 119, 91-112 (2016)

24. Banerjee, P., Surendran, H., Chowdhury, D. R., Prabhakar, K. \& Pal, R. Metformin mediated reversal of epithelial to mesenchymal transition is triggered by epigenetic changes in E-cadherin promoter. J. Mol. Med. 94, 1397-1409 (2016).

25. Tsukada, T. et al. Transforming growth factor beta 1 induces apoptotic cell death in cultured human umbilical vein endothelial cells with down-regulated expression of bcl-2. Biochem. Biophys. Res. Commun. 210, 1076-1082 (1995).

26. Ziello, J. E., Jovin, I. S. \& Huang, Y. Hypoxia-Inducible Factor (HIF)-1 regulaton pathway and its potential for therapeutic intervention in malignancy and ischemia. Yale J. Biol. Med. 80, 51-60 (2007).

27. Liu, C. J. et al. Transcriptional activation of cartilage oligomeric matrix protein by Sox9, Sox5, and Sox6 transcription factors and CBP/p300 coactivators. Front. Biosci. 12, 3899-3910 (2007).

28. Huang, J. \& Guo, L. Knockdown of SOX9 inhibits the proliferation, invasion, and EMT in thyroid cancer cells. Oncol. Res. 25, 167-176 (2017).

29. Bodar, E. J., Simon, A. \& van der Meer, J. W. Effects of the histone deacetylase inhibitor ITF2357 in autoinflammatory syndromes. Mol. Med. 17, 363-368 (2011).

30. Carta, S. et al. Histone deacetylase inhibitors prevent exocytosis of interleukin1 beta-containing secretory lysosomes: role of microtubules. Blood $\mathbf{1 0 8}$, 1618-1626 (2006).

31. Lewis, E. C. et al. The oral histone deacetylase inhibitor ITF2357 reduces cytokines and protects islet beta cells in vivo and in vitro. Mol. Med. 17, 369-377 (2011).

32. Fan, Z. \& Guan, J. Antifibrotic therapies to control cardiac fibrosis. Biomater. Res. 20, 13 (2016).

33. Lim, R. R. et al. ITF2357 transactivates Id3 and regulate TGFbeta/BMP7 signaling pathways to attenuate corneal fibrosis. Sci. Rep. 6, 20841 (2016).

34. Siciliano, C. et al. The potential of GMP-compliant platelet lysate to induce a permissive state for cardiovascular transdifferentiation in human mediastinal adipose tissue-derived mesenchymal stem cells. Biomed. Res. Int. 2015, 162439 (2015).

35. Euler, G. Good and bad sides of TGFbeta-signaling in myocardial infarction. Front Physiol. 6, 66 (2015).

36. Antos, C. L. et al. Dose-dependent blockade to cardiomyocyte hypertrophy by histone deacetylase inhibitors. J. Biol. Chem. 278, 28930-28937 (2003).

37. Bolden, J. E. et al. HDAC inhibitors induce tumor-cell-selective pro-apoptotic transcriptional responses. Cell Death Dis. 4, e519 (2013).

38. Kern, S., Feng, H. Z., Wei, H., Cala, S. \& Jin, J. P. Up-regulation of alpha-smooth muscle actin in cardiomyocytes from non-hypertrophic and non-failing transgenic mouse hearts expressing $\mathrm{N}$-terminal truncated cardiac troponin I. FEBS Open Bio. 4, 11-17 (2013).

39. Lamouille, S., Xu, J. \& Derynck, R. Molecular mechanisms of epithelialmesenchymal transition. Nat. Rev. Mol. Cell. Biol. 15, 178-196 (2014).

40. Kanisicak, O. et al. Genetic lineage tracing defines myofibroblast origin and function in the injured heart. Nat. Commun. 7, 12260 (2016).

41. Bearzi, C. et al. PIGF-MMP9-engineered iPS cells supported on a PEGfibrinogen hydrogel scaffold possess an enhanced capacity to repair damaged myocardium. Cell Death Dis. 5, e1053 (2014).

42. Nural-Guvener, H., Zakharova, L., Feehery, L., Sljukic, S. \& Gaballa, M. Anti-fibrotic effects of class I HDAC inhibitor, mocetinostat is associated with |L-6/ Stat3 signaling in ischemic heart failure. Int. J. Mol. Sci. 16, 11482-11499 (2015).

43. Zhang, L. et al. Inhibition of histone deacetylases preserves myocardial performance and prevents cardiac remodeling through stimulation of endogenous angiomyogenesis. J. Pharmacol. Exp. Ther. 341, 285-293 (2012).

44. Rizzi, R. et al. Post-natal cardiomyocytes can generate iPS cells with an enhanced capacity toward cardiomyogenic re-differentation. Cell Death Differ 19. 1162-1174 (2012) 